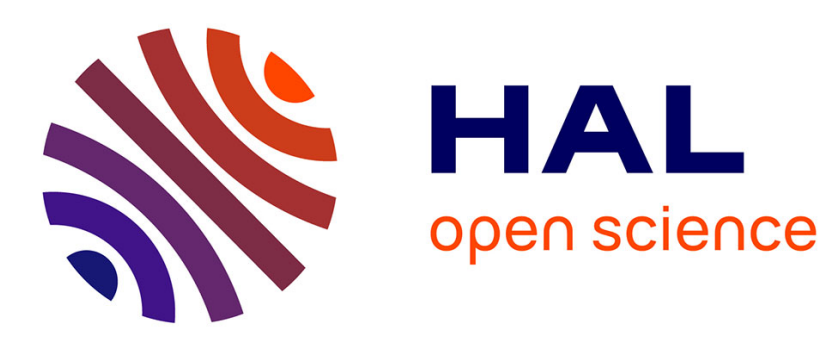

\title{
Studying the Inter-Relation Between Locomotion Techniques and Embodiment in Virtual Reality
}

\author{
Diane Dewez, Ludovic Hoyet, Anatole Lécuyer, Ferran Argelaguet
}

\section{To cite this version:}

Diane Dewez, Ludovic Hoyet, Anatole Lécuyer, Ferran Argelaguet. Studying the Inter-Relation Between Locomotion Techniques and Embodiment in Virtual Reality. IEEE International Symposium on Mixed and Augmented Reality (ISMAR), 2020, Arrecife, Brazil. pp.1-10. hal-03008902

\section{HAL Id: hal-03008902 https://hal.science/hal-03008902}

Submitted on 17 Nov 2020

HAL is a multi-disciplinary open access archive for the deposit and dissemination of scientific research documents, whether they are published or not. The documents may come from teaching and research institutions in France or abroad, or from public or private research centers.
L'archive ouverte pluridisciplinaire HAL, est destinée au dépôt et à la diffusion de documents scientifiques de niveau recherche, publiés ou non, émanant des établissements d'enseignement et de recherche français ou étrangers, des laboratoires publics ou privés. 


\title{
Studying the Inter-Relation Between Locomotion Techniques and Embodiment in Virtual Reality
}

\author{
Diane Dewez* \\ Ludovic Hoyet $^{\dagger} \quad$ Anatole Lécuyer ${ }^{\dagger}$ \\ Inria, Univ Rennes, CNRS, IRISA, France
}

Ferran Argelaguet ${ }^{\S}$
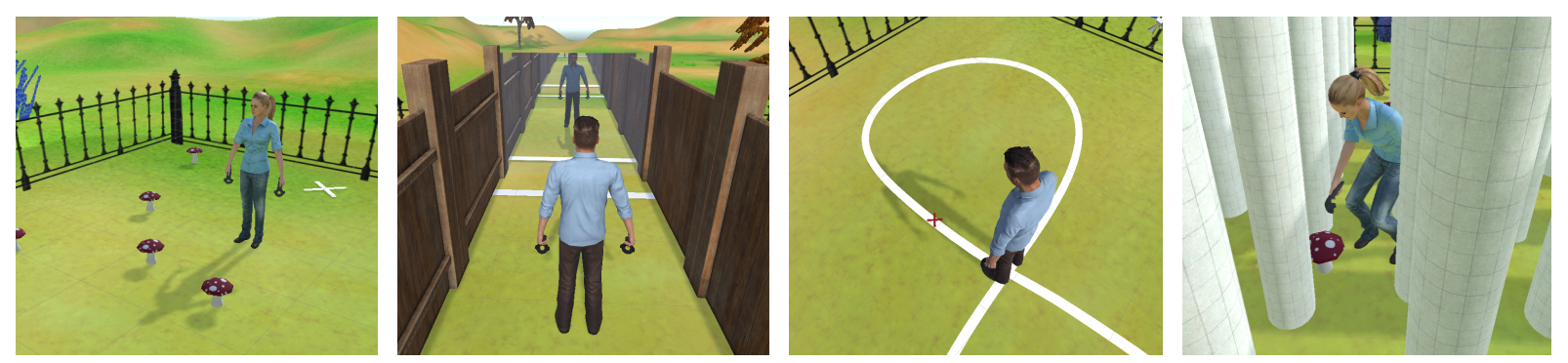

Figure 1: Illustration of the four tasks performed by the participants in our user study, all in the full-body avatar condition. The same tasks were also performed without an avatar. From left to right: Training task, Corridor task, Path-following task and Columns task.

\begin{abstract}
Virtual embodiment and navigation are two topics widely studied in the virtual reality community. Despite the potential inter-relation between embodiment and locomotion, studies on virtual navigation rarely supply users with a virtual representation, while studies on virtual embodiment rarely allow users to virtually navigate. This paper therefore explores this potential inter-relation by focusing on the two following questions: Does the locomotion technique have an impact on the user's sense of embodiment? Does embodying an avatar have an impact on the user's preference and performance depending on the locomotion technique?

To address these questions, we conducted a user study $(N=60)$ exploring the relationship between the locomotion technique and the user's sense of embodiment over a virtual avatar seen from a first-person perspective. Three widely used locomotion techniques were evaluated: real walking, walking-in-place and virtual steering. All participants performed four different tasks involving a different awareness of their virtual avatar. Participants also performed the same tasks without being embodied in an avatar. The results show that participants had a comparable sense of embodiment with all techniques when embodied in an avatar, and that the presence or absence of the virtual avatar did not alter their performance while navigating, independently of the technique. Taken together, our results represent a first attempt to qualify the inter-relation between virtual navigation and virtual embodiment, and suggest that the $3 \mathrm{D}$ locomotion technique used has little influence on the user's sense of embodiment in VR.
\end{abstract}

Index Terms: Human-centered computing-Human computer interaction (HCI) - HCI design and evaluation methods-User studies; Human-centered computing-Human computer interaction (HCI)Interaction paradigms-Virtual reality

\footnotetext{
*e-mail: diane.dewez@inria.fr

$\dagger$ e-mail: ludovic.hoyet@inria.fr

†e-mail: anatole.lecuyer@inria.fr

$\S$ e-mail: ferran.argelaguet@inria.fr
}

\section{INTRODUCTION}

Locomotion in immersive virtual environments is essential, as it provides a way to explore an environment or to reach interactable objects. However, unlike in real life, we cannot walk infinitely in a virtual environment, as we are limited by the tracking space. Many techniques have therefore been imagined to tackle this limitation [2]. Some of these techniques use virtual movement (i.e. virtual steering) or physical motions but with lower interaction fidelity (i.e. walkingin-place), i.e. which are not similar to the way we interact in real life [33].

While these techniques have proved their efficiency, they have however mostly been studied without the use of full-body avatars. Embodying an avatar have proven beneficial, for example to improve spatial awareness [13]. As their use in virtual environments becomes more and more common, it also seems crucial to study the potential impact of having a virtual body on locomotion. In particular, several questions arise in relation to the use of avatars with existing locomotion techniques. Because previous work showed that locomotion techniques involving physical movement can enhance the sense of presence [50], we first ask the question: can different locomotion techniques elicit different levels of "virtual embodiment" [22] (i.e., the appropriation of the avatar)? Also, the presence of a virtual avatar was also shown to influence the perception of affordances [31], obstacle avoidance [37] and perception of redirected walking [27]. We therefore ask the second question: does embodying an avatar have an impact on a user's preference and performance depending on the locomotion technique?

To explore these effects, we conducted a mixed-design experiment in virtual reality. We used three widely used locomotion techniques as a between-subject factor: real walking, walking-inplace and head steering. We chose these techniques because they are all continuous locomotion techniques, with different levels of physical engagement and interaction fidelity. Participants performed four tasks in the virtual environment, with two conditions on their virtual appearance (within-subject factor): full-body animated avatar or only 3D models of controllers. The results showed that, with the chosen tasks, participants had a comparable sense of embodiment with all techniques, and that the presence or absence of their virtual avatar did not alter their performance while navigating. Still, they generally preferred the condition with a full-body avatar, except in the steering condition where opinions were more divided. 
The remainder of the paper is structured as follows. First, related work on avatars and navigation is presented in Section 2. Then, Section 3 details the experiment performed to explore both the influence of the avatar on locomotion and the effect of three locomotion techniques on the sense of embodiment. Section 4 describes the results of the experiment, which are discussed in Section 5. Finally, Section 6 provides the concluding remarks.

\section{Related Work}

This section presents previous work which studied navigation and avatars. First, we review some general papers on navigation. Then a few works which used avatars in their experiment are reviewed, some of which explored the potential influence of avatars on navigation. Finally, we present related work on the sense of embodiment which contained a navigation task.

\subsection{Navigation in Virtual Environments}

Navigation in virtual reality has been widely studied [2], and many techniques have been created to tackle the problem of limited physical space, like walking-in-place [47] or teleportation [9]. A recent taxonomy classified these locomotion techniques into four categories: room-scale-based (natural walking), motion-based (e.g. walking-in-place), teleport-based and controller-based (like the different types of steering) [8].

As most studies are typically technology-oriented, and mostly focus on user preference or performance with a given technique, they seldom evaluate the effect of the presence of an avatar. Few experiments have added a virtual body during navigation tasks, but often with the goal of enhancing the sense of presence or because a virtual body was necessary to the implementation of the proposed locomotion technique.

\subsubsection{Avatars in Navigation Tasks}

A minority of studies on navigation used a virtual body in their implementation, in part because of the additional work involved in terms of implementation in the past. For instance, Slater et al. [46] added a virtual body in their virtual treadmill metaphor with the goal of enhancing presence. However, the avatar was quite simple: only the user's right hand and head were tracked, and the rotation of the virtual body depended on the head rotation. They used a similar tracking with a more elaborated avatar a few years later in their study on the influence of the locomotion technique on presence [50], which showed that walking elicited a higher sense of presence than walking-in-place or flying. Other works also included an avatar because of experimental requirements, e.g., with locomotion techniques implying the use of a third-person perspective [40], or to improve spatial awareness, e.g., by displaying feet to climb stairs [39].

\subsubsection{Effects of Having an Avatar on Navigation}

Recently, some works started exploring the impact of having a virtual representation during navigation. For instance, Kruse et al. [27] explored the influence of displaying feet in the virtual environments on perception of translation gains during redirected-walking. While no effect of the feet was found, the environment richness seemed to have an influence on the perception of translational gains. However, feet can still be important for spatial perception, as it was found that changing feet size can change the way we perceive a gap width as well as our capacity of stepping over this gap [18].

Similarly, McManus et al. [34] found that people presented with a virtual body performed better in a stepping stone task. This task necessitating a good spatial awareness, the virtual body was helping participants to know their location in the virtual environment, and to easily step on the stones. It was also recently found that people had a greater tendency to avoid an obstacle when they had a full-body avatar [42]. In the context of affordances, Lin et al. [31] showed that the presence of an avatar changed the users' perception of being able to step over a pole, but had no significant influence on their decision to pass through a doorway, as recently confirmed by Bhargava et al. [6]. The choice of rotating shoulders to pass an aperture and avoid collisions was found influenced by the presence of an avatar [37]. Similarly, the avatar's gender can also have an influence on affordances, in particular in collaborative virtual environments. Buck et al. [11] studied two-person joint actions while passing through apertures, and found an effect of avatar's gender on participants' behaviour. However, these behaviours are a bit different and more complex than the ones observed in real-life.

Recently, Park et al. [43] studied the influence of the visual selfrepresentation on user's perception during walking-in-place. In a preliminary study, they tested two types of feedback (visual walkingin-place or natural walking). People reported a preference for the natural walking animation, although it would sometimes display unintended steps.

While more and more experiments therefore consider the effects of displaying avatars, there is still no strong focus on the users' perception, in terms of acceptance of the virtual representation. Do they prefer to have an avatar? Do they easily embody their avatar during navigation? Does embodiment affect navigation? Embodiment is a recent concept that needs to be studied when using navigation, especially because embodiment plays a role in our behaviour, e.g., on how we interact with virtual objects [21], or on how we perceive virtual object sizes [4].

\subsection{Studies on Embodiment Using Navigation Tasks}

The sense of embodiment towards a body B has been defined as "the sense that emerges when B's properties are processed as if they were the properties of one's own biological body." [22]. It is often divided into three subcomponents: body ownership, agency and self-location. While in most experiments on the sense of embodiment the user tends to stay at the same virtual position in the virtual environment $[3,4]$, some experiments involved the user to navigate in the virtual environment as is the focus of this paper. For instance, some studies explored whether participants can feel embodiment towards a walking avatar while remaining seated: Leonardis et al. [30] found that providing vestibular and proprioceptive feedback could increase the sense of embodiment; as for Kokkinara et al. [25], they found that people felt agency over the walking with a first-person perspective, but not with a third-person perspective. Similar results were obtained by a study from Galvan Debarba et al. [15] which found that without actually walking, participants could experience ownership over a virtual body which walks, yet, they experienced a lower sense of agency than when really walking. In contrast, when walking, they showed that a sense of embodiment could be felt over the walking avatar even with third-person perspective. The influence of both perspective and avatar realism in a navigation task was also studied by Medeiros et al. [35]. In their study, the virtual representation was influencing the navigation behaviour: with the most realistic appearance, participants' paths were smoother and involved less collisions. Other works have also explored other input control mechanisms, for example Cohen et al. [12] assessed the sense of embodiment experienced when making an avatar walk using a Brain-Computer Interface. Finally, Koilias et al. [24] showed that the sense of embodiment, and in particular the sense of agency, can be influenced by motion artifacts. Interestingly, the impact of motion artifacts on agency is strongly dependent on the observation point of view, where agency is more negatively affected by motion artifacts when users are facing a mirror than when they are looking down at themselves walking.

While these studies have not explored the influence of the locomotion technique on the sense of embodiment, some recent studies have begun to take into account the link between locomotion and embodiment. For instance, Oberdörfer et al. [41] studied the special 
case of transition techniques and found no significant effect of the technique on body ownership over a wooden manikin. Embodiment is also beginning to be considered as an evaluation criterion when implementing new locomotion techniques [36]. This paper therefore studies embodiment while using different locomotion techniques.

\section{EXPERIMENT}

Our general aim was to explore the implications of having an avatar while navigating in a virtual environment (VE). The first goal was to study the potential differences between the locomotion techniques in terms of perception of the avatar, mostly regarding embodiment, i.e. whether different locomotion techniques can elicit different levels of "virtual embodiment". The second goal was to compare locomotion techniques with and without a virtual body, which can influence both user preference and performance. In this study, participants therefore performed several navigation tasks with and without a virtual body, using one of these techniques: real walking, walking-in-place or head steering. We collected quantitative data as well as answers to several subjective questionnaires.

\subsection{Participants}

Sixty-five participants volunteered to take part in our experiment. The majority of them were students and staff from our research center. Five participants were removed from the study: two because of problems in data recording, two because of tracking issues and one because of motion sickness during the first block. In the end, sixty participants (age $\min =20, \max =56$, avg $=28.9 \pm 8.4,25$ women and $35 \mathrm{men}$ ) took part in our study. Participants did not receive any compensation. They were all naive as to the purpose of the experiment, and gave written and informed consent. The study conformed to the declaration of Helsinki. Twenty-five participants reported having no or little previous experience in VR (score 1 or 2 out of 7), twenty-four to have some previous experience in VR (score between 3 and 5) and eleven to be experts (score 6 or 7 out of 7).

\subsection{Apparatus}

The experiment was developed using Unity (version 2019.1.0f2). Users were tracked using an HTC Vive PRO HMD (equipped with the HTC wireless adapter so that participants were not hindered by the cable when navigating), two HTC Vive controllers, two HTC Vive trackers positioned on the ankles to track the feet, and two HTC Vive trackers positioned on a backpack to track the shoulders. We used four HTC Vive lighthouses to track an $8 \mathrm{~m} \times 8 \mathrm{~m}$ area. The virtual environment consisted of a flat grassy ground surrounded by fences, matching the dimensions of the physical space, with trees and hills in the background (see Figure 2).

The positions of the headset, controllers and trackers on the ankles were used to animate the avatar, using inverse kinematics (FinalIK plugin). Participants were represented by gender-matched avatars (visible in Figure 1). During calibration, participants were asked to keep their feet and legs straight to adjust the avatar's feet. They also had to stand straight to adjust the avatar's global scale based on their height.

\subsection{Locomotion Techniques}

To better understand the influence of the presence of an avatar depending on the locomotion technique, we selected different locomotion techniques based on Boletsis's taxonomy [8]: room-scale-based, motion-based, controller-based and teleportation-based. In particular, we were interested in techniques that are commonly used in virtual reality, and that involved a similar motion in the VE but different levels of physical movement. Therefore, we chose the following techniques: Real Walking (RW; room-scale-based), WalkingIn-Place (WIP; motion-based) and Steering (S; controller-based). Despite its inclusion in Boletsis's taxonomy, we did not include

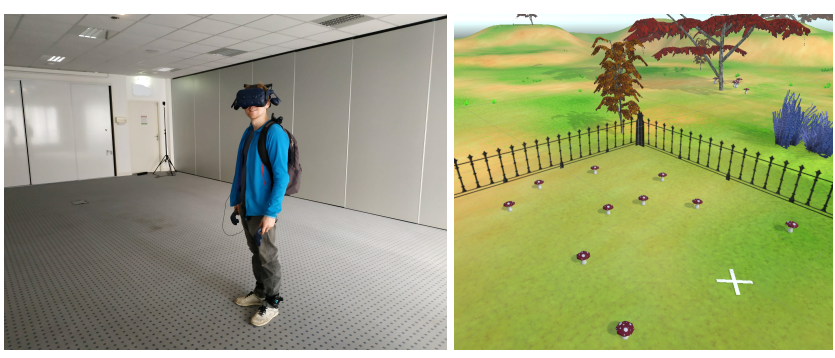

Figure 2: Left: Setup in the physical environment. The HTC Vive with a wireless adapter was used to enable participants to physically walk in an $8 \mathrm{mx} 8 \mathrm{~m}$ area. The tracking of the user's motions was enabled by the two hand controllers and two HTC Vive Trackers attached to their ankles. Two additionnal HTC Vive trackers were also positioned on a backpack to track participants' shoulders. Tracking was done using four HTC Vive lighthouses. Right: Overview of the virtual environment: the navigation area was constrained by the virtual fences which matched the limits of the physical space.

teleportation in our study as it creates a discontinuous movement unlike the three other selected techniques. The different techniques are detailed below:

- Real Walking (RW): Participants could physically walk in an $8 \mathrm{~m} \times 8 \mathrm{~m}$ area. A 1:1 control mapping was kept between the physical and the virtual motions.

- Walking-In-Place (WIP): we implemented a WIP algorithm which detects walking patterns based on the position of the ankles [10,23], which were tracked using Vive trackers. Locomotion was initiated whenever a first step was detected, i.e., when the two ankles' position had consecutively passed above a given threshold (initial tracker position $+5 \mathrm{~cm}$ ). It was stopped whenever a step had not been detected in the last $0.8 \mathrm{~s}$. Because participants tend to take lower steps over time, the threshold was decreased to a value equal to the initial tracker position $+2 \mathrm{~cm}$ after they started stepping. The thresholds were determined empirically through iterative design and several user tests. The direction was determined by the participant's head orientation.

- Steering (S): in this technique, participants navigated in the virtual environment using a constant navigation speed while keeping the Vive controller button held down, which is a common approach in both VR and video games. We chose a head-steering implementation (i.e., navigation direction determined by the participant's head orientation), as it is commonly used, easy to get familiar with, and because the tasks in our experiment did not require looking in another direction than the one in which people were headed.

While previous work identified that people usually walk in real situations at a comfort speed of approximately $1.3 \mathrm{~m} / \mathrm{s}$ [7], several studies demonstrated that participants tend to walk slower in an immersive VE $[1,5,38]$, especially when wearing an HMD. We therefore set the navigation speed of both the Steering and WalkingIn-Place techniques to $1.0 \mathrm{~m} / \mathrm{s}$, which should approximately match the speed observed while walking with an HMD $[1,5]$.

At this point, it also seems important to point out that for all three techniques in our experiment, the avatar's movements were always driven by the participants' actual movements when they were embodied in a virtual avatar. This means that a) for the real walking technique, the avatar walked as the participant physically walked; b) for the walking-in-place technique, the avatar performed steps on the spot like the participant, while being translated forward in 
the direction in which participants were looking; c) for the virtual steering, since participants did not move forward in the real world, the avatar stood like the participant, moved its feet like the participant when they turned on the spot, or "slid" along the ground in the direction participants were looking towards.

\subsection{Experimental Tasks}

The experiment was divided into four tasks (see Figure 1), which were designed to ensure that participants were aware of their virtual body, i.e. parts of the avatar were directly visible from the participant's perspective. The main design principle for defining the tasks was to ensure that participants were able to see different parts of their virtual body during the experiment in a consistent way.

- Training task: The first task was a training task, which goal was to get participants used to the virtual environment and the locomotion technique. More precisely, participants were instructed to navigate in the virtual environment, in order to pick mushrooms on the ground by touching them with either hand. This task lasted one minute.

- Corridor task: The aim of this task was to enable participants to see their entire avatar moving, while navigating in a virtual environment. They were therefore instructed to walk back and forth in a corridor with mirrors at both ends. A line in front of the mirror indicated where subjects were to turn around so as to not go through the mirror. This task lasted two minutes.

- Path-following task: The aim of this task was to force participants to look at their avatar, in particular at their feet, while navigating. They were therefore instructed to follow a target moving on an eight-shaped trajectory, both displayed at the ground level. The target was always $50 \mathrm{~cm}$ ahead of them so that participants had to tilt their head, thus ensuring that the avatar's legs were inside their field of view. This task lasted two minutes.

- Columns task: The aim of this task was to study people's behaviour while navigating around virtual obstacles. They were instructed to navigate in a virtual environment filled with 2-metre high columns and to pick mushrooms as in the training task. In this task, people could therefore mostly see their hands and arms. The mushrooms were always placed at the same positions. They were displayed in groups of ten, with new mushrooms appearing once all those of a series were picked. The distance between columns was 1.45 times the shoulder width of the scaled avatar, to ensure that any participant could move through without necessarily turning their shoulders (which requires an aperture-to-shoulder ratio lower than $1.4[37,51])$. This task lasted two minutes.

The tasks were always presented in the same order: training, corridor, path-following and columns. As we were interested in evaluating the overall users' subjective experience, we considered that keeping the same order would reduce the variability in their subjective assessment. Furthermore, we did not compare the tasks with one another.

\subsection{Experimental Protocol and Design}

First, participants signed the consent form. Then they were equipped with the HMD, the controllers and the trackers.

We had two independent variables: Technique and Appearance. For the Technique, we used a between-subjects design, so each participant used only one locomotion technique. The three techniques used were presented in Section 3.3. There were twenty participants per group. For the Appearance condition, a within-subject design was chosen. Two levels were used: Full-Body Avatar (FBA) and No
Avatar (NA). In the FBA condition, participants were represented by a full-body gender-matched avatar holding a 3D model of an HTC Vive controller in each hand, while in the NA condition, only the 3D models of the Vive controllers were displayed.

Therefore, the experiment was divided into two blocks (FBA and NA). In total, participants performed $2 \times 4$ tasks. To minimize potential ordering effects, the blocks were counterbalanced and there was a 5-minute break between them. Participants therefore either performed all the four tasks (in the order presented above) in the FBA condition, then all four tasks in the NA condition, or the opposite (see Table 1). The whole session lasted about 40 minutes, including the participant's welcome, performing the experiment, and answering questionnaires.

As dependent variables, we used both objective (performance) and subjective (user preference, embodiment) criteria.

\subsubsection{Objective Data}

Objective data were measured in only two tasks (path-following and columns tasks). No objective data were recorded during the training task as the aim of the task was only to make users familiar with the given locomotion technique. Similarly, there were no relevant performance criteria in the corridor task.

- Path-following task: The mean distance between the path to follow and the user's position was computed as an objective measure of performance.

- Columns task: We measured both the number of mushrooms picked during the columns task, and the number of collisions with the columns, as an objective measure of performance. Because the number of collisions can also depend on the distance covered by participants, we normalized the number of collisions by the distance covered (in metres). We counted collisions between the participants' shoulders (tracked by the additional Vive trackers) and columns hereafter referred as shoulder collisions.

\subsubsection{Subjective Data}

Subjective questionnaires were also used to obtain user perception of the experience.

Embodiment questionnaire: We used the ownership, agency and self-location questions from the embodiment questionnaire proposed by Gonzalez-Franco and Peck [16] (see Table 2). These questions were evaluated on a 7-point Likert scale from 1 (Strongly disagree) to 7 (Strongly agree). The questionnaire was administered only after the FBA block.

Representation questionnaire: After each block, participants were asked questions related to the virtual representation (R questions in Table 3). These questions were about both the virtual representation seen indirectly in the mirror and in other tasks from the first-person perspective. The goals of these questions were mainly to detect whether people were disturbed by their representation $\left(R_{\text {MirrorLogical }}\right.$ and $\left.R_{\text {OtherLogical }}\right)$ and whether they appreciated it ( $R_{\text {MirrorPleasing }}$ and $\left.R_{\text {OtherPleasing }}\right)$. Each item was measured on a 7-point Likert scale. Participants answered these questions once after each block.

Comparison questionnaire: Participants were also asked to answer several additional questions after the last block, related to whether they preferred the presence of an avatar or not (see Table 4). Again, questions were rated on a 7-point Likert scale. Participants could also associate an adjective with each Appearance condition, explaining why they preferred to have an avatar or not, as well as make free comments about the experiment. 
SSQ: Additionally, the Simulator Sickness Questionnaire (SSQ) [20] was given to participants before and after the experiment, and we computed the difference between pre-experiment and post-experiment scores to verify that there were no differences in terms of sickness between the different conditions.

\subsection{Hypotheses}

Previous studies have shown the impact of physical implication on the sense of embodiment [48]. Moving in the VE seems to let the brain compare the movement intention with the feedback of the action, which is one of the ways to increase the sense of agency [49] if there is a match. This sense of agency can strengthen the sense of ownership [19]. Thus, we expected that the higher the match between proprioception and sensory feedback, the higher the felt embodiment would be, leading to the following hypothesis:

H1 The sense of embodiment is influenced by the locomotion technique. In particular, we expected the sense of embodiment to be higher for techniques with a higher interaction fidelity (i.e., $\mathrm{RW}>\mathrm{WIP}>\mathrm{S}$ ).

In addition, because an avatar provides a better spatial awareness [13], we expected that participants would avoid the columns more in the columns task with the FBA condition. Participants could also see the columns as a threat for their avatar. Thus our second main hypothesis was:

H2 The presence of an avatar leads to less collisions with the virtual environment.

Furthermore, we also included secondary hypotheses, related to the other collected variables:

H3 People find having a full-body avatar while navigating in a VE more pleasing and logical (R questions).

H4 People think that their representation is more disturbing when using WIP and S than RW (R questions).

\section{ANALYSIS}

Mixed two-way ANOVA analyses were used taking into account both the Technique (between-subjects) and the Appearance (withinsubjects) factors. We tested the normality assumption using the Shapiro-Wilk normality test. When the data were not normal, we applied an Aligned Rank Transformation (ART) on the data before performing the ANOVA analysis. Tukey HSD post-hoc tests $(\alpha=.05)$ were conducted to check significance for pairwise comparisons. Kruskal-Wallis tests were however conducted when analysing results depending only on the Technique (between-subjects), as the ShapiroWilk tests significantly rejected the null hypothesis according to which the data were normally distributed. Dunn's post-hoc tests were conducted to perform pairwise comparisons.

\subsection{Effect of the Locomotion Technique on Embodi- ment}

First of all, we used a Mann-Whitney's test to identify potential ordering effects between the FBA and NA conditions. As we did not find any significant effect, order will not be considered in the remainder of the analysis.

We performed Kruskal-Wallis tests to explore differences in embodiment ratings between the different techniques, using the questions about the senses of ownership, agency and self-location. We only found a significant effect of the Technique on the question $S L_{\text {located }}$ about self-location $\left(\chi^{2}=9.623, p<0.01\right)$. Dunn's test revealed that the RW condition obtained significantly higher scores than WIP and $\mathrm{S}$ conditions (see values in Table 2). We did not find any significant difference between any of the three techniques for all the other questions. These results therefore do not support H1. Medians and quartiles for each question are available in Table 2. Although not significantly different, the scores for the questions $O_{\text {someoneElse }}$ and $O_{\text {someoneElseMirror }}$ were higher for the RW condition. We also computed the overall ownership, agency and self-location ratings using the recommendations from Gonzalez Franco and Peck [16], and did not find any significant difference. Overall, the agency ratings $(M=5.00, S D=0.81)$ as well as self-location ratings $(M=5.43, S D=1.09)$ were high. For ownership, the scores were slightly lower $(M=4.60$, $S D=1.21$.

\subsection{Users' Performance and Collision Avoidance}

We performed a mixed two-way ANOVA to study the potential differences between the different techniques (between-subjects factor) and the avatar conditions (within-subjects factor) on the objective measures of the path-following (mean distance to trajectory) and columns task ( shoulder collisions, number of collected mushrooms).

We did not find any main effect of the Appearance or interaction effects on these objective measures (see Table 5). In particular, this result does not support $\mathbf{H 2}$ (no influence on the number of collisions). These results were also confirmed by participants' answers to the subjective questions about the ease of tasks (questions $C_{\text {pathEasier }}$ and $C_{\text {columnsEasier }}$ in Table 4 , which results are detailed in Section 4.3.2). Participants did not find the task easier in one or the other Appearance condition, which is coherent with the objective data.

\subsection{Users' Preferences}

We also performed a mixed two-way ANOVA to explore the differences in the subjective answers between the different conditions (Technique and Appearance). Some questions were meant to indirectly compare appearance between the two blocks, while others were meant to directly ask participants which condition they preferred at the end of the experiment.

\subsubsection{Representation questionnaire}

For the questions about the virtual representation, we found a main effect of Appearance for the question $R_{\text {MirrorLogical }}\left(F_{1,57}=4.48, p<\right.$ $0.05)$. Post-hoc tests showed that participants felt that being embodied in an avatar was more logical $(M d n=5, I Q R=3-6)$ than without $(M d n=$ $4, I Q R=3-6)$. We also found an effect of Appearance for the question $R_{\text {OtherPleasing }}\left(F_{1,57}=9.341, p<0.01\right)$. Only the results of $R_{\text {MirrorLogical }}$ and $R_{\text {OtherPleasing }}$ support $\mathbf{H 3}$.

Also, we observed that participants provided lower ratings for the $R_{\text {MirrorLogical }}$ and $R_{\text {MirrorPleasing }}$ questions compared to $R_{\text {OtherLogical }}$ and $R_{\text {OtherPleasing }}$ respectively. Being in front of the mirror seemed to have a negative impact on the participants' answers.

No effect of the locomotion Technique was found on questions about representation, which does not support $\mathbf{H 4}$.

\subsubsection{Comparison questionnaire}

Regarding the questions directly asking participants to compare conditions, the results show a slight preference for the condition with an avatar, with all medians $\geq 4$ for $C_{\text {preferBody }}$. Yet, we observed a polarization for $S$ (see Figure 3), some participants not at all preferring having a virtual body $(\leq 2)$, and others showing a high preference $(=7)$. These results were not visible for the WIP and RW conditions in which participants provided more consistent answers with a slight preference over the avatar. Regarding the questions asking to compare the level of ease between the two blocks (see Table $4, C_{\text {pathEasier }}$ and $C_{\text {columnsEasier }}$ ), participants did not find the path-following and column tasks to be easier in one condition or another (answers around 4), independently of the Technique. 
Table 1: The two possible orderings of the experiment based on the avatar factor. While the representation questionnaire was administered after each block, the embodiment questionnaire was only administered after the FBA condition.

1) SSQ
\begin{tabular}{|c|c|c|c|c|c|c|}
\hline Full-Body Avatar & Rep. + Embodiment & No Avatar & SSQ & Rep. & Comparison \\
\hline 2) SSQ & No Avatar & Rep. & Full-Body Avatar & SSQ & Rep. + Embodiment & Comparison \\
\hline
\end{tabular}

Table 2: Summary for body ownership (O), agency (A) and self-location (SL) questionnaires. This table reports the median and interquartile range for each question. When significant differences (Kruskal-Wallis test) were found between techniques (NW, WIP, S), the descriptive statistics for each technique are provided.

\begin{tabular}{|c|c|c|c|c|}
\hline \multirow{2}{*}{ ID } & \multirow{2}{*}{ Questions } & \multicolumn{3}{|c|}{ Median[Q1;Q3] } \\
\hline & & NW & WIP & $\mathrm{S}$ \\
\hline OwnBody & I felt as if the virtual body was my own body & \multicolumn{3}{|c|}{$5[3 ; 6]$} \\
\hline$O_{\text {someoneElse }}$ & I felt as if the virtual body I saw was someone else & \multicolumn{3}{|c|}{$4[2 ; 5]$} \\
\hline OmoreOneBody & It seemed as if I might have more than one body & \multicolumn{3}{|c|}{$2[1 ; 3]$} \\
\hline OwnBodyMirror & I felt as if the virtual body I saw when looking in the mirror was my own body & \multicolumn{3}{|c|}{$4[2.75 ; 5.25]$} \\
\hline$O_{\text {someoneElseMirror }}$ & I felt as if the virtual body I saw when looking at myself in the mirror was another person & \multicolumn{3}{|c|}{$3[2 ; 5]$} \\
\hline$A_{\text {control }}$ & It felt like I could control the virtual body as if it was my own body & \multicolumn{3}{|c|}{$5[5 ; 6]$} \\
\hline$A_{\text {caused }}$ & The movements of the virtual body were caused by my movements & \multicolumn{3}{|c|}{$6[5 ; 7]$} \\
\hline$A_{\text {influencing }}$ & I felt as if the movements of the virtual body were influencing my own movements & \multicolumn{3}{|c|}{$3[2 ; 5]$} \\
\hline$A_{\text {movingItself }}$ & I felt as if the virtual body was moving by itself & \multicolumn{3}{|c|}{$2[1 ; 3]$} \\
\hline SLlocated & I felt as if my body was located where I saw the virtual body & $6[6 ; 7]^{1}$ & $5[4 ; 6]^{2}$ & $5[4 ; 6]^{2}$ \\
\hline$S L_{\text {outBody }}$ & I felt out of my body & \multicolumn{3}{|c|}{$2[1 ; 4]$} \\
\hline
\end{tabular}

Techniques sharing a superscript were not significantly different (Tukey HSD $\alpha=.05$ )

Table 3: Summary for subjective questions on the virtual representation (R). This table reports the median and interquartile range for each question. When main effects of appearance factor or interaction effects were found, the descriptive statistics for each appearance are provided.

\begin{tabular}{|c|c|c|c|}
\hline \multirow[b]{2}{*}{ ID } & \multirow[b]{2}{*}{ Questions } & \multicolumn{2}{|c|}{ Median[Q1;Q3] } \\
\hline & & FBA & NA \\
\hline$R_{\text {MirrorLogical }}$ & When facing the mirror, my virtual representation when navigating in the VE was confusing(1)/logical(7) & $5[3 ; 6]$ & $4[3 ; 6]$ \\
\hline RotherLogical & In other tasks, my virtual representation when navigating in the VE was confusing $(1) / \operatorname{logical}(7)$ & \multicolumn{2}{|c|}{$6[4 ; 6.25]$} \\
\hline RirrorPleasing & When facing the mirror, my virtual representation when navigating in the VE was disturbing(1)/pleasing(7) & \multicolumn{2}{|c|}{$4[3 ; 5]$} \\
\hline RotherPleasing & In other tasks, my virtual representation when navigating in the VE was disturbing(1)/pleasing(7) & $5[4 ; 6]$ & $4[4 ; 5.25]$ \\
\hline
\end{tabular}

* indicates that an interaction effect was found

Table 4: Summary for comparison questionnaire. This table reports the median and interquartile range for each question. There was no significant effect (Kruskal-Wallis test) of the Technique factor.

\begin{tabular}{|c|c|c|c|c|}
\hline \multirow{2}{*}{ ID } & \multirow{2}{*}{ Questions } & \multicolumn{3}{|c|}{ Median[Q1;Q3] } \\
\hline & & NW & WIP & $\mathrm{S}$ \\
\hline$C_{\text {preferBody }}$ & I preferred having a body vs. not having a body & \multicolumn{3}{|c|}{$5[3 ; 6.25]$} \\
\hline$C_{\text {pathEasier }}$ & I felt that performing the path-following task was easier with an avatar vs. without an avatar & \multicolumn{3}{|c|}{$4[3 ; 6]$} \\
\hline$C_{\text {columnsEasier }}$ & I felt that performing the columns task was easier with an avatar vs. without an avatar & \multicolumn{3}{|c|}{$4[4 ; 5]$} \\
\hline C behaviourDifferent & I have the impression that my behaviour was different with an avatar vs. without an avatar & \multicolumn{3}{|c|}{$5[4 ; 6]$} \\
\hline
\end{tabular}

Table 5: Performance results: mean distance to trajectory (in $\mathrm{cm}$ ), ratio of shoulder collisions, and number of picked mushrooms

\begin{tabular}{c|c|c}
\hline & FBA & NA \\
\hline Mean distance & $M=8.14, S D=2.96$ & $M=8.50, S D=2.91$ \\
Ratio shoulder collisions & $M=0.35, S D=0.41$ & $M=0.37, S D=0.38$ \\
Nb mushrooms & $M=22.7, S D=7.13$ & $M=23.45, S D=6.41$
\end{tabular}

\subsection{Additional Analyses}

While we did not find that the presence of an avatar affected the number of collisions with the environment, previous studies have shown that people being embodied in an avatar usually tend to avoid obstacles more in the virtual environment $[37,42]$. Therefore, we decided to explore this effect in more details by analyzing whether the number of collisions could be influenced by the level of embodiment reported by participants. In order to investigate this effect, we computed the correlations (Spearman) between the embodiment questionnaire and the amount of collisions in the FBA condition (as we did not ask embodiment questions in the NA condition). Regarding self-location questions, we found a significant negative correlation between the question $S L_{\text {located }}$ and the ratio of shoulder collisions $(r=-0.31, p<0.05)$. We did not find similar correlations for the questions about ownership or agency. This result suggests that 


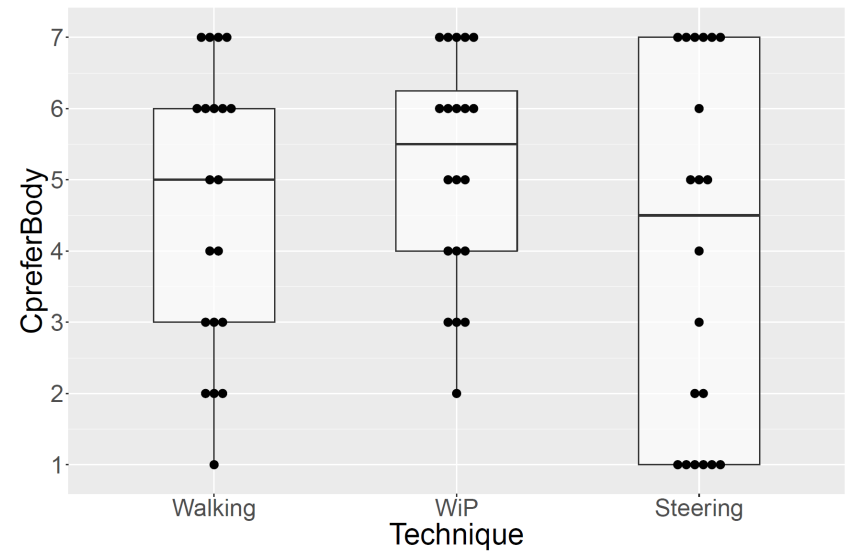

Figure 3: Scores by Technique for the question $C_{\text {preferBody }}$; no significant difference between the techniques but a high dispersion for the $\mathrm{S}$ condition.

people who felt that they were co-located with their virtual body collided less with the environment. Variations in cybersickness scores over the course of the experiment were not significantly different between techniques $\left(\chi^{2}=0.87, p=0.65\right)$.

\section{Discussion}

This study explored the potential inter-effects between the use of locomotion techniques and avatars. The first goal was to study the effect of the locomotion technique on embodiment, while the second goal was to explore the impact of the presence of an avatar during navigation, depending on the technique. In this section, we discuss the results on the sense of embodiment,collision avoidance, and finally user preference.

\subsection{Embodiment}

Our first main hypothesis was that the choice of a locomotion technique would influence the sense of embodiment. In particular, we expected high-fidelity locomotion techniques, requiring more physical movements, to elicit a higher sense of embodiment, similarly to previous results on the sense of presence $[47,50]$. Contrary to our expectations, there was no significant difference on the level of embodiment between the locomotion techniques, suggesting that the choice of a technique does not influence the participants' sense of embodiment.

Two potential reasons may explain this result. A first potential explanation is that participants had a total control over their avatar movements, no matter what technique they used, which has been shown to play a primary role in the sense of embodiment [14]. For all tasks, when participants moved, the avatar moved accordingly, therefore there was always a coherence between participants' movements and the feedback. Even for the steering technique, participants had to reorient their body to perform the tasks and were aware of the upper-body motions (e.g. holding the controller in front of them, picking mushrooms). Even though participants were mostly stationary when using the walking-in-place and steering techniques, they still felt a high sense of embodiment, which is consistent with studies in which people embodied avatars even though they were not moving $[15,25]$. However, contrary to related studies [25], the avatar was not necessarily walking in these conditions (especially with the steering technique) in our study. Having an avatar sliding on the ground did not seem to affect participants' sense of embodiment.

A second explanation of our results could be that visual incongruities due to animation quality might have slightly disturbed par- ticipants. Even though the difference was not significant, we noticed slightly lower ownership scores in the walking condition, which would be in contradiction with our hypothesis. Because it is the most realistic and high-fidelity technique of locomotion, people may have more expectations in terms of matching between the visual feedback and their proprioception. It is known that the match between visual and motor signals plays an important role in inducing a sense of ownership [26], and that motion artifacts can affect the sense of agency while walking [24].

In summary, our experiment suggests that total control of avatar movements might contribute more to embodiment than the coherence between avatar movements and camera motion, although artifacts or differences between participants' real movements and the avatar's movements can modulate their sense of embodiment. Further studies will be required to better assess the contribution of the upper-body and lower-body control, as well as the impact of animation artifacts on the sense of embodiment.

\subsection{Collision Avoidance}

Our second main hypothesis was that people would collide less with the columns when embodied in a full-body avatar, however it was not supported by the results. In general, we observed different behaviours among participants, some avoided obstacles while others went through them. Participants who ignored the columns reported that they did it to pick the mushrooms faster, and because they were not disturbed by going through them, contrary to people who avoided them. This result differs from other studies that have found that people tended to avoid obstacles when they were embodied in a realistic avatar, but these studies used obstacles on the trajectory of the hand [3] or at lower height [42]. We believe that the task and the obstacles could explain the difference in our results, as the avatar may have not contributed to avoid the columns. First, the task required participants to focus on finding and picking the mushrooms which could have diverted their attention. Second, the obstacles might not have been close enough to compel the participants to use a body reference to avoid collisions.

\subsection{Preference}

\subsubsection{Logical and pleasing aspects}

We expected that people would find the condition with a full-body avatar more logical and pleasing. For the logical aspect, this was the case for the task involving a mirror. However, some participants reported that the avatar's movements were sometimes "disturbing", for example that the knees would bend too much compared to what they were doing. The absence of an avatar was still perceived as less logical, where several participants reported negatively about seeing only controllers in the mirror condition. Participants did not find it significantly more logical to have a full-body avatar in other tasks. This may be due to the fact that most VR applications currently only use floating hands or controllers as virtual representations, and that participants were therefore not surprised by this simpler representation (controllers only). For the pleasing aspect, the results were significant only for the tasks not involving the mirror. Although participants appreciated having a body visible from a first-person perspective, they did not find it more pleasing to see the avatar in the mirror. A potential explanation could be that several participants reported being disturbed by the difference in external appearance (clothes, haircut, morphology) of the avatar compared to their own, yielding lower scores on this question.

\subsubsection{Preferred condition}

There were several questions asking participants whether they preferred the condition with or without an avatar. In particular, we expected that participants would prefer the condition with an avatar, and would find the navigation tasks easier to perform in such cases. The high variance in the answers to the question "I preferred having 
a body" shows different categories of people. Some participants were really disturbed by an absence of body and preferred having an avatar. Such participants described the experience with adjectives like "realistic" or "immersive" to describe the condition with an avatar. Other subjects did not prefer any of the conditions, or tended to prefer the condition without an avatar, as the avatar could be seen as "an obstacle" or too different from them that it was more disturbing than helping. This could potentially be explained by them being more task-oriented. They described this condition with adjectives like "easy", "transparent", "unconstrained" or "less disturbing".

The most used words for the condition with an avatar were: funny (9), easy (8), disturbing (7), realistic (6), immersive (5) and interesting (5). For the condition without avatar, it was: easy (9), simple (6), interesting (5) and disturbing (5). While adjectives for the condition without an avatar were more focused on tasks achievement (easy, simple), it is interesting to point that positive adjectives related to immersion and realism only appeared in the condition with an avatar However, while our results show a slight preference for having an avatar in the navigation tasks of our experiment, it is possible that this preference is not directly related to the task but that it might be common to virtual experiences. It would therefore be interesting to explore potential effects of the task on this preference in future studies.

\subsubsection{Ease of tasks}

Similarly to the work by Lugrin et al. [32], the presence of a virtual body to perform the tasks did not seem to impact the user in terms of performance or preference. Similarly to their study using an action-based game, our experiment contained goal-oriented tasks, especially in the columns task. In particular, some participants of our experiment reported that they only needed the controllers to perform the tasks. They therefore seemed to have been more focused on achieving the task, possibly reducing the awareness of the presence of the avatar. For tasks where they had to notice the avatar (mirror task and path-following task mostly), some participants considered the avatar as an obstacle or an annoyance that was diverting them from the task. Our results need to be considered relatively to the tasks, and other tasks could be tested. For example, tasks which need a body reference and a finer control of the avatar, like the stepping task in the work by McManus et al. [34], could be investigated.

\subsection{Limitations and Future Work}

One of the limitation of this study is related to the degree of realism of the avatars and their animation. For instance, the calibration only adjusted the global scale of the avatar, while other papers studying avatars and collisions used more precise calibrations [37]. Several solutions are now starting to appear to easily and precisely calibrate an avatar to the participants morphology [44], which could help in the future to more accurately measure collisions with the environment. In terms of animation, several participants were also disturbed by the leg movements, especially when physically walking in front of the mirror. It was previously found that offset rotation of joints can affect embodiment [24]. It could therefore be interesting to run a similar study with higher-quality motion capture systems to evaluate whether differences in terms of embodiment could be influenced by the animation quality in such cases. Still, animating avatars with Inverse Kinematics as performed in this study is a common procedure in such embodiment studies $[17,45]$. Similarly, it would be interesting to investigate different levels of avatar realism, as it was found to influence embodiment [28], and to explore potential inter-effects with the locomotion technique.

Even though people felt a sense of embodiment while using walking-in-place and steering techniques, some participants noticed that the feedback was strange, especially in front of the mirror. As future work, it would be interesting to study different types of visual feedbacks for the techniques. As an example, several studies have started to compare different feedbacks for the walking-in-place technique $[29,43]$, which can be walking animation, synchronous stepping or running animation. Different types of avatar animation could lead to different levels of embodiment, with the goal of finding one optimal feedback for each locomotion technique that would guarantee logic, naturalness and embodiment. Other locomotion techniques might also be studied. For instance, this paper did not focus on teleportation because, unlike the three techniques evaluated, it does not imply a continuous movement. It would be interesting to study this commonly used technique with an avatar, as Oberdörfer et al. [41] started with a wooden manikin, e.g., by studying different effects or types of avatar animation in combination with teleportation.

\section{Conclusion}

The study presented in this paper explored the inter-relation between avatar embodiment and locomotion techniques. The study considered three locomotion techniques requiring different user involvement (walking, stepping in place, limited physical movement) and three main tasks involving a different awareness of the virtual avatar that participants had full control of. Overall, we found that people experienced similar levels of embodiment with the three locomotion techniques and that the coherence of the avatar's motion with respect to motion of the virtual environment did not create any disruption in the sense of embodiment. Our results represent a first attempt to qualify the inter-relation between locomotion and virtual embodiment, and suggest that the locomotion technique used has little influence on the user's sense of embodiment in VR when the user has a full control of his avatar movements.

\section{ACKNOWLEDGMENTS}

We wish to thank all the reviewers for their constructive comments, as well as the participants who took part in our experiment. This work was sponsored by the Inria Research Challenge Avatar.

\section{REFERENCES}

[1] P. Agethen, V. S. Sekar, F. Gaisbauer, T. Pfeiffer, M. Otto, and E. Rukzio. Behavior analysis of human locomotion in the real world and virtual reality for the manufacturing industry. ACM Transactions on Applied Perception (TAP), 15(3):20, 2018.

[2] M. Al Zayer, P. MacNeilage, and E. Folmer. Virtual locomotion: A survey. IEEE Transactions on Visualization and Computer Graphics, 26(6):2315-2334, 2020.

[3] F. Argelaguet, L. Hoyet, M. Trico, and A. Lecuyer. The role of interaction in virtual embodiment: Effects of the virtual hand representation. In 2016 IEEE Virtual Reality (VR), pp. 3-10, 2016. doi: 10.1109/VR. 2016.7504682

[4] D. Banakou, R. Groten, and M. Slater. Illusory ownership of a virtual child body causes overestimation of object sizes and implicit attitude changes. Proceedings of the National Academy of Sciences, 110(31):12846-12851, 2013. doi: 10.1073/pnas. 1306779110

[5] F. Berton, A. Olivier, J. Bruneau, L. Hoyet, and J. Pettre. Studying gaze behaviour during collision avoidance with a virtual walker: Influence of the virtual reality setup. In 2019 IEEE Conference on Virtual Reality and 3D User Interfaces (VR), pp. 717-725, 2019.

[6] A. Bhargava, H. Solini, K. Lucaites, J. W. Bertrand, A. Robb, C. C. Pagano, and S. V. Babu. Comparative evaluation of viewing and self-representation on passability affordances to a realistic sliding doorway in real and immersive virtual environments. In 2020 IEEE Conference on Virtual Reality and $3 D$ User Interfaces (VR), pp. 519-528, 2020. doi: 10.1109/VR46266.2020.00-3

[7] R. W. Bohannon. Comfortable and maximum walking speed of adults aged 20-79 years: reference values and determinants. Age and ageing, 26(1):15-19, 1997.

[8] C. Boletsis. The new era of virtual reality locomotion: A systematic literature review of techniques and a proposed typology. Multimodal Technologies and Interaction, 1:24, 2017. doi: 10.3390/mti1040024 
[9] E. Bozgeyikli, A. Raij, S. Katkoori, and R. Dubey. Point \& teleport locomotion technique for virtual reality. In Proceedings of the 2016 Annual Symposium on Computer-Human Interaction in Play, CHI PLAY '16, pp. 205-216, 2016. doi: 10.1145/2967934.2968105

[10] L. Bruno, J. Madeiras Pereira, and J. Jorge. A new approach to walking in place. 09 2013. doi: 10.1007/978-3-642-40477-1_23

[11] L. E. Buck, J. J. Rieser, G. Narasimham, and B. Bodenheimer. Interpersonal affordances and social dynamics in collaborative immersive virtual environments: Passing together through apertures. IEEE Transactions on Visualization and Computer Graphics, 25(5):2123-2133, 2019.

[12] O. Cohen, M. Koppel, R. Malach, and D. Friedman. Controlling an avatar by thought using real-time fMRI. Journal of neural engineering, 11(3):035006, 2014.

[13] M. H. Draper, M. J. Wells, V. J. Gawron, and I. Tom A. Furness. Exploring the influence of a virtual body on spatial awareness. Proceedings of the Human Factors and Ergonomics Society Annual Meeting, 40(22):1146-1150, 1996.

[14] R. Fribourg, F. Argelaguet, A. Lécuyer, and L. Hoyet. Avatar and sense of embodiment: Studying the relative preference between appearance, control and point of view. IEEE Transactions on Visualization and Computer Graphics, 26(5):2062-2072, May 2020. doi: 10.1109/TVCG .2020 .2973077

[15] H. Galvan Debarba, S. Bovet, R. Salomon, O. Blanke, B. Herbelin, and R. Boulic. Characterizing first and third person viewpoints and their alternation for embodied interaction in virtual reality. PLOS ONE, 12(12):1-19, 2017. doi: 10.1371/journal.pone.0190109

[16] M. González-Franco and T. C. Peck. Avatar embodiment. towards a standardized questionnaire. Frontiers in Robotics and AI, 5:74, 2018. doi: 10.3389/frobt.2018.00074

[17] M. González-Franco, D. Pérez-Marcos, B. Spanlang, and M. Slater. The contribution of real-time mirror reflections of motor actions on virtual body ownership in an immersive virtual environment. In 2010 IEEE Virtual Reality Conference (VR), pp. 111-114, 2010. doi: 10. 1109/VR.2010.5444805

[18] E. Jun, J. K. Stefanucci, S. H. Creem-Regehr, M. N. Geuss, and W. B. Thompson. Big foot: Using the size of a virtual foot to scale gap width. ACM Trans. Appl. Percept., 12(4):16:1-16:12, 2015. doi: 10. $1145 / 2811266$

[19] A. Kalckert and H. Ehrsson. Moving a rubber hand that feels like your own: A dissociation of ownership and agency. Frontiers in human neuroscience, 6:40, 2012. doi: 10.3389/fnhum.2012.00040

[20] R. S. Kennedy, N. E. Lane, K. S. Berbaum, and M. G. Lilienthal Simulator sickness questionnaire: An enhanced method for quantifying simulator sickness. The international journal of aviation psychology, 3(3):203-220, 1993.

[21] K. Kilteni, I. Bergstrom, and M. Slater. Drumming in immersive virtual reality: The body shapes the way we play. IEEE Transactions on Visualization and Computer Graphics, 19(4):597-605, 2013. doi: 10.1109/TVCG.2013.29

[22] K. Kilteni, R. Groten, and M. Slater. The sense of embodiment in virtual reality. Presence: Teleoperators and Virtual Environments, 21(4):373-387, 2012. doi: 10.1162/PRES_a_00124

[23] J. Kim, D. Gračanin, and F. Quek. Sensor-fusion walking-in-place interaction technique using mobile devices. In 2012 IEEE Virtual Reality Workshops (VRW), pp. 39-42, 2012

[24] A. Koilias, C. Mousas, and C.-N. Anagnostopoulos. The effects of motion artifacts on self-avatar agency. Informatics, 6(2), 2019. doi: 10 3390/informatics6020018

[25] E. Kokkinara, K. Kilteni, K. J. Blom, and M. Slater. First person perspective of seated participants over a walking virtual body leads to illusory agency over the walking. Scientific reports, 6:28879, 2016.

[26] E. Kokkinara and M. Slater. Measuring the effects through time of the influence of visuomotor and visuotactile synchronous stimulation on a virtual body ownership illusion. Perception, 43(1):43-58, 2014. PMID: 24689131. doi: $10.1068 / \mathrm{p} 7545$

[27] L. Kruse, E. Langbehn, and F. Steinicke. I can see on my feet while walking: Sensitivity to translation gains with visible feet. In 2018 IEEE Conference on Virtual Reality and 3D User Interfaces (VR), pp. 305-312, 2018. doi: 10.1109/VR.2018.8446216
[28] M. E. Latoschik, D. Roth, D. Gall, J. Achenbach, T. Waltemate, and M. Botsch. The effect of avatar realism in immersive social virtual realities. Proceedings of the 23rd ACM Symposium on Virtual Reality Software and Technology, 2017.

[29] J. Lee, M. Lee, G. J. Kim, and J.-I. Hwang. Effects of virtual gait visualization in walk-in-place on body ownership and presence. In Extended Abstracts of the 2020 CHI Conference on Human Factors in Computing Systems Extended Abstracts, CHI '20, p. 1-7. Association for Computing Machinery, New York, NY, USA, 2020. doi: 10.1145/ 3334480.3382867

[30] D. Leonardis, A. Frisoli, M. Barsotti, M. Carrozzino, and M. Bergamasco. Multisensory feedback can enhance embodiment within an enriched virtual walking scenario. Presence: Teleoperators and Virtual Environments, 23(3):253-266, 2014.

[31] Q. Lin, J. Rieser, and B. Bodenheimer. Stepping over and ducking under: The influence of an avatar on locomotion in an hmd-based immersive virtual environment. In Proceedings of the ACM Symposium on Applied Perception, SAP '12, pp. 7-10, 2012. doi: 10.1145/2338676 .2338678

[32] J.-L. Lugrin, M. Ertl, P. Krop, R. Klüpfel, S. Stierstorfer, B. Weisz, M. Rück, J. Schmitt, N. Schmidt, and M. E. Latoschik. Any "body" there? avatar visibility effects in a virtual reality game. In 2018 IEEE Conference on Virtual Reality and 3D User Interfaces (VR), pp. 17-24, 2018. doi: 10.1109/VR.2018.8446229

[33] R. P. McMahan, D. A. Bowman, D. J. Zielinski, and R. B. Brady. Evaluating display fidelity and interaction fidelity in a virtual reality game. IEEE Transactions on Visualization and Computer Graphics, 18(4):626-633, 2012.

[34] E. A. McManus, B. Bodenheimer, S. Streuber, S. de la Rosa, H. H. Bülthoff, and B. J. Mohler. The influence of avatar (self and character) animations on distance estimation, object interaction and locomotion in immersive virtual environments. In Proceedings of the ACM SIGGRAPH Symposium on Applied Perception in Graphics and Visualization, APGV '11, pp. 37-44, 2011. doi: 10.1145/2077451. 2077458

[35] D. Medeiros, R. K. dos Anjos, D. Mendes, J. a. M. Pereira, A. Raposo, and J. Jorge. Keep my head on my shoulders!: Why third-person is bad for navigation in vr. In Proceedings of the 24th ACM Symposium on Virtual Reality Software and Technology, VRST '18, pp. 16:1-16:10, 2018. doi: $10.1145 / 3281505.3281511$

[36] D. Medeiros, A. Sousa, A. Raposo, and J. Jorge. Magic carpet: Interaction fidelity for flying in vr. IEEE Transactions on Visualization and Computer Graphics, 2019.

[37] D. R. Mestre, C. Louison, and F. Ferlay. The contribution of a virtual self and vibrotactile feedback to walking through virtual apertures. In M. Kurosu, ed., Human-Computer Interaction. Interaction Platforms and Techniques, pp. 222-232. Springer International Publishing, 2016.

[38] B. Mohler, J. Campos, M. Weyel, and H. H. Bülthoff. Gait parameters while walking in a head-mounted display virtual environment and the real world. In B. Fröhlich, R. Blach, and R. van Liere, eds., 13th Eurographics Symposium on Virtual Environments and 10th Immersive Projection Technology Workshop (IPT-EGVE 2007), pp. 85-88. Eurographics Association, 2007.

[39] R. Nagao, K. Matsumoto, T. Narumi, T. Tanikawa, and M. Hirose. Ascending and descending in virtual reality: Simple and safe system using passive haptics. IEEE Transactions on Visualization and Computer Graphics, 24(4):1584-1593, 2018. doi: 10.1109/TVCG.2018. 2793038

[40] N. Navarro Griffin and E. Folmer. Out-of-body locomotion: Vectionless navigation with a continuous avatar representation. In Proceedings of ACM Conference (Conference'17). ACM, 2019.

[41] S. Oberdörfer, M. Fischbach, and M. Latoschik. Effects of VE Transition Techniques on Presence, Illusion of Virtual Body Ownership, Efficiency, and Naturalness. In Proceedings of the Symposium on Spatial User Interaction, pp. 89-99, 2018. doi: 10.1145/3267782.3267787

[42] Y. Pan and A. Steed. How foot tracking matters: The impact of an animated self-avatar on interaction, embodiment and presence in shared virtual environments. Frontiers in Robotics and AI, 6:104, 2019. doi: 10.3389/frobt.2019.00104

[43] C. Park and K. Jang. Investigation of visual self-representation for a 
walking-in-place navigation system in virtual reality. In 2019 IEEE Conference on Virtual Reality and 3D User Interfaces (VR), pp. 11141115. IEEE, 2019.

[44] S. Pujades, B. Mohler, A. Thaler, J. Tesch, N. Mahmood, N. Hesse, H. H. Bülthoff, and M. J. Black. The virtual caliper: Rapid creation of metrically accurate avatars from $3 \mathrm{~d}$ measurements. IEEE Transactions on Visualization and Computer Graphics, 25(5):1887-1897, 2019. doi: 10.1109/TVCG.2019.2898748

[45] D. Roth, J. Lugrin, J. Büser, G. Bente, A. Fuhrmann, and M. E. Latoschik. A simplified inverse kinematic approach for embodied vr applications. In 2016 IEEE Virtual Reality (VR), pp. 275-276, 2016. doi: 10.1109/VR.2016.7504760

[46] M. Slater, A. Steed, and M. Usoh. The virtual treadmill: A naturalistic metaphor for navigation in immersive virtual environments. First Eurographics Workshop on Virtual Reality, pp. 71-86, 1993.

[47] M. Slater, M. Usoh, and A. Steed. Taking steps: the influence of a walking technique on presence in virtual reality. ACM Transactions on Computer-Human Interaction (TOCHI), 2(3):201-219, 1995.

[48] M. Synofzik, G. Vosgerau, and A. Newen. I move, therefore i am: A new theoretical framework to investigate agency and ownership. Consciousness and Cognition, 17(2):411 - 424, 2008. Social Cognition, Emotion, and Self-Consciousness.

[49] M. Synofzik, G. Vosgerau, and M. Voss. The experience of agency: an interplay between prediction and postdiction. Frontiers in Psychology, 4:127, 2013. doi: 10.3389/fpsyg.2013.00127

[50] M. Usoh, K. Arthur, M. C. Whitton, R. Bastos, A. Steed, M. Slater, and F. P. Brooks Jr. Walking $>$ walking-in-place $>$ flying, in virtual environments. In Proceedings of the 26th annual conference on Computer graphics and interactive techniques, pp. 359-364. ACM Press/Addison-Wesley Publishing Co., 1999.

[51] W. Warren and S. Whang. Visual guidance of walking through apertures: Body-scaled information for affordances. Journal of experimental psychology. Human perception and performance, 13:371-83, 1987. doi: 10.1037/0096-1523.13.3.371 